\title{
Quand la pénibilité du travail s'invite à la maison
}

Perspectives féministes en santé au travail

\section{Christelle Avril et Pascal Marichalar}

\section{(2) OpenEdition}

\section{Journals}

Édition électronique

URL : http://journals.openedition.org/travailemploi/7110

DOI : $10.4000 /$ travailemploi. 7110

ISSN : 1775-416X

Éditeur

DARES - Ministère du Travail

Édition imprimée

Date de publication : 1 juillet 2016

Pagination : 5-26

ISSN : 0224-4365

Référence électronique

Christelle Avril et Pascal Marichalar, «Quand la pénibilité du travail s'invite à la maison », Travail et Emploi [En ligne], 147 | juillet-septembre 2016, mis en ligne le 11 juillet 2019, consulté le 24 septembre 2020. URL : http://journals.openedition.org/travailemploi/7110 ; DOI : https://doi.org/10.4000/ travailemploi. 7110 


\title{
Introduction
}

\section{Quand la pénibilité du travail s'invite à la maison Perspectives féministes en santé au travail ${ }^{*}$}

\author{
Christelle Avril $^{* *}$, Pascal Marichalar ${ }^{* * *}$
}

$\mathrm{E}$ n 1700, le médecin italien Bernardino Ramazzini publie un livre qui sera traduit en français une dizaine d'années avant la Révolution : l'Essai sur les maladies des artisans. Il est reconnu comme le premier ouvrage présentant de manière systématique les problèmes de santé rencontrés en fonction du métier exercé. Parmi les cinquante-deux chapitres, seuls trois sont consacrés à l'examen de métiers exclusivement féminins : les sages-femmes, les nourrices et les blanchisseuses (ces « femmes toujours dans des lieux humides, ayant les pieds et les mains continuellement mouillés ») (RAMAZZINI, 1777, p. 335). Le médecin évoque également les femmes qui occupent la fonction de portefaix, les religieuses, les paysannes qui s'occupent du rouissage du chanvre et du lin dans les mares, ou encore les travailleuses de la soie - s'étonnant du fait que ces dernières soient toutes des femmes, «comme si la nature n'avait formé la soie que pour leur usage » (ibid., p. 344).

Bien plus proche de nous, le développement, à partir des années 1970, de recherches en sciences sociales autour des problèmes de conditions de travail et de santé a réactivé certains des questionnements, mais aussi des impensés, de l'approche du professeur de médecine de Modène et de Padoue. Dans cette introduction au dossier «Quand la pénibilité du travail s'invite à la maison », nous revenons sur la manière dont les différences de conditions de travail entre femmes et hommes ont été prises en compte dans les sciences sociales au cours des quatre dernières décennies. Nous distinguons trois moments - logiques plutôt que strictement chronologiques - dans la perspective féministe sur ces questions. Dans un premier temps, nous présentons le constat des différences sexuées des risques et des pénibilités au travail ; pour saisir

\footnotetext{
* Les auteurs tiennent à remercier Magali Marcille, Thomas Amossé, Céline Bessière, l'ensemble du comité de rédaction de Travail et Emploi, ainsi que les évaluateurs extérieurs pour leurs relectures critiques et constructives de ce texte ou des autres textes du dossier.

** Institut de recherche interdisciplinaire sur les enjeux sociaux (Iris ; UMR 8156), École des hautes études en sciences sociales (EHESS) ; christelle.avril@ehess.fr.

*** Iris, Centre national de la recherche scientifique (CNRS) ; pascal.marichalar@cnrs.fr.
} 
ensuite les enjeux de la critique du caractère genré des données sur la santé au travail, ainsi que des politiques de prévention ; enfin, dans un troisième temps, nous mettons l'accent sur l'intérêt d'étudier conjointement travail rémunéré et travail domestique afin de renouveler ce domaine d'étude. Les quatre articles qui composent le présent dossier sont nourris des apports des deux premiers moments, et amorcent l'étude du troisième, apportant nous semble-t-il un éclairage nouveau et des résultats en propre dans un domaine qui reste cependant encore largement à défricher.

Dès la première enquête statistique française sur les conditions de travail de 1978, des chercheurs questionnent la variable sexe et montrent que femmes et hommes ne sont pas exposés aux mêmes risques professionnels (Molinié, VolKOFF, 1980). Ils ouvrent la voie à la mise en évidence de ce que l'on peut appeler la division sexuée des pénibilités et des risques professionnels. Ce constat s'accompagne dans les études statistiques françaises d'une réflexion sur la ségrégation professionnelle, soit le phénomène qui fait que la plupart des femmes et des hommes travaillent dans des professions qui sont très majoritairement peuplées par des membres du même sexe qu'elles et eux (HuEt, 1983)².

À partir de la fin des années 1970, de nouvelles analyses en termes de genre tendent à déconstruire la neutralité de la catégorie du sexe. Dans l'étude du travail, le caractère naturel des compétences réputées féminines ou masculines perd de son évidence : « Lorsque les employeurs ont qualifié de travail des femmes des opérations mécaniques et répétitives, ils n'ont pas seulement renforcé des stéréotypes existant déjà sur la nature des femmes, ils ont aussi contribué à de nouvelles définitions de la féminité » (TILLY, SCOTT, 1987, pp. 16-17). Une approche inédite de l'étude sexuée des problèmes de santé au travail se développe, en particulier au Canada, et est progressivement introduite en Europe à partir du début des années 2000. En France, des revues de sociologie comme Travail, genre et sociétés et Les Cahiers du genre, ou encore la revue Travailler ${ }^{3}$, spécialisée en psychopathologie et en psychodynamique du travail, ainsi que des chercheuses comme Annie Thébaud-Mony jouent le rôle de passeuses ${ }^{4}$. Selon cette perspective, il ne suffit pas de mettre au jour la répartition sexuée des pénibilités et des risques, comme on se cantonne encore parfois à le faire en France. Les sciences sociales ont également intérêt à questionner la construction des données et notamment la visibilité différentielle des risques et de leurs conséquences en fonction du genre. Cette posture critique interroge plus spécifiquement le fait que les hommes occuperaient toujours des postes plus dangereux ou pénibles que ceux des femmes. Contrairement à ce que laissent entendre de récentes dispositions réglementaires en

1. Le terme de « ségrégation professionnelle », sans plus de précision, renvoie dans les publications à la ségrégation sexuée ou genrée dans le monde du travail.

2. Pour une synthèse des approches genrées dans la statistique française, voir FouQUET (2003).

3. Ces trois revues ont notamment publié à plusieurs reprises des articles de Karen Messing (encadré) et de son équipe au début des années 2000.

4. Annie Thébaud-Mony (2000) mais aussi Jennifer BuÉ (2004) intègrent respectivement les contraintes familiales ou encore la division sexuée des conditions de travail dans leurs analyses en santé au travail. 
matière de médecine du travail et de départ à la retraite, les « postes à risque » ou les « facteurs de pénibilité » ne sont des conditions majoritairement masculines que dans la mesure où l'on continue d'ignorer et de minimiser les contraintes rencontrées par les femmes dans leur travail.

Il reste cependant un angle mort dans la prise en compte critique du genre dans les études des problèmes de santé liés au travail. Il s'agit de l'étude de l'articulation entre le travail rémunéré (ce que les enquêtes officielles entendent généralement par travail) et le travail non rémunéré (aussi appelé travail domestique), qui permet de mieux comprendre à la fois la spécificité des risques sanitaires auxquels femmes et hommes sont exposés, et les différences de nature et de visibilité des dommages qu'ils engendrent. Cette perspective s'appuie sur les acquis de décennies de recherches féministes portant à la fois sur l'inégale répartition du travail domestique et sur le rôle invisible des femmes dans la carrière de leur conjoint - un soutien dont elles ne bénéficient généralement pas elles-mêmes. En esquissant une étude conjointe aussi bien de l'univers familial que de l'univers du travail, ce dossier nous semble montrer l'intérêt d'analyser l'imbrication complexe des facteurs de genre et des facteurs de pénibilité. Il rappelle avec force, contre une approche de la santé au travail qui se centrerait uniquement sur le monde du travail, qu'il est simpliste d'opposer la famille réparatrice au travail dangereux : le travail peut être dangereux mais aussi émancipateur, en particulier pour les femmes (LE FEUVRE, 2014). La famille peut protéger tout comme elle peut être elle-même déstabilisée et exposée aux pénibilités du travail. Elle peut aussi, et enfin, aggraver les inégalités de conditions de travail et de pénibilités entre femmes et hommes.

Notre utilisation du terme « féministe » en étonnera certains sans doute, tant il est malheureusement rabattu, en France, sur une approche qui ne parviendrait pas à se départir d'objectifs politiques catégoriels et qui verserait dans le «non-scientifique » (LAGRAVE, 1990). Notre intention est autre. Si l'on sait depuis longtemps que la reconnaissance des atteintes à la santé par le travail est le produit de luttes politiques (Lenoir, 1980 ; Daubas-LetourneuX, Thébaud-Mony, 2001) et que les données les plus objectives en apparence sont toujours le fruit de combats historiques (ROSENTAL, DeVINCK, 2007 ; HATZFELD, 2008), une perspective féministe sur la santé au travail invite à regarder au-delà des seuls rapports de classe. Elle montre que la production de savoir sur les expériences des femmes et des hommes au travail a été historiquement nourrie, et continue de l'être, par des combats indissociablement scientifiques et politiques dont l'un des enjeux est de rappeler que le domaine privé est aussi politique et que les données les plus « objectives » intègrent des biais de genre ${ }^{5}$. Elle dévoile les biais de construction des données, la manière dont les risques sont naturalisés et définis comme acceptables, ainsi que les logiques pseudo-scientifiques qui conduisent

5. En statistique, cet angle de recherche est suggéré par Michel GollaC (1997). De manière générale, des sociologues (ForTino, 2014 ; BERCOT, 2014 ; GousSARD, 2014 ; BERCOT, 2015) et des historiennes (TurnER, 2014) ont également depuis peu ouvert ce chantier. Un numéro récent de la revue Raison présente réunit de nombreuses contributions critiques sur le thème « Genre et santé au travail » (PFEFFERKORN, PoLESI, 2014). 
à borner les frontières de ce qui est défini (ou pas) comme maladie professionnelle. En cela, la perspective féministe sur la santé au travail n'est en rien une perspective spécifique aux femmes. Un regard féministe sur la santé au travail permet de mettre au jour des mécanismes généraux de production des inégalités de santé au travail et établit des connaissances qui valent pour l'ensemble des travailleurs.

\section{La division sexuée des pénibilités et des risques}

La première enquête statistique sur les conditions de travail effectuée par ce qui s'appelait alors le Service des études et de la statistique (SES) du ministère du Travail et de la Participation date de 1978 ; la dernière a été réalisée par la Direction de l'animation de la recherche, des études et des statistiques (Dares) en 2013. Depuis vingt ans, deux autres grandes enquêtes ont également été mises en place : l'enquête Surveillance médicale des expositions des salariés aux risques professionnels (Sumer) depuis 1994 et l'enquête Santé et itinéraire professionnel (SIP) depuis 2006. Toutes ces enquêtes ont permis de rendre visible la division sexuée des pénibilités dans le monde du travail (Guignon, 2008 ; Bouffartigue et al., 2010 ; Amossé, Daubas-Letourneux, 2012).

Dès la première enquête française sur les conditions de travail, une attention particulière a été portée aux différences entre femmes et hommes. Dans un article pionnier sur les ouvrières et les ouvriers, Anne-Françoise Molinié et Serge Volkoff montrent qu'en 1978 les femmes ont des horaires plus fixes que les hommes et sont davantage contrôlées par une pointeuse. Elles ont un travail plus répétitif : «Plus d'une OS [ouvrière spécialisée] femme sur quatre travaille à la chaîne, alors que ce type de travail ne concerne qu'un OS homme sur treize » (Molinié, Volkoff, 1980, p. 35). Leur rémunération est plus souvent liée à leur rythme de travail. Enfin, «plus de femmes que d'hommes sont en butte à une stricte interdiction de converser avec leurs collègues » (ibid., p. 38). De telles conclusions sur le monde ouvrier sont également mises en évidence par des approches qualitatives réalisées à la même époque (KERGOAT, 1978) et restent en grande partie d'actualité dans les décennies qui suivent (Gollac, Volkoff, 2002).

Les enquêtes récentes établissent que les hommes sont plus confrontés aux efforts physiques et aux contraintes posturales, et à des environnements de travail nuisibles ou dangereux (saleté, humidité, courants d'air, températures élevées, températures basses, etc.) : $53 \%$ des hommes déclarent rester debout longtemps, contre 48,5\% des femmes, et $44,8 \%$ des hommes affirment porter ou déplacer des charges lourdes, contre $37,1 \%$ des femmes ${ }^{6}$. Les hommes sont également plus exposés aux risques professionnels tels que respirer des fumées et des poussières, être en contact avec des produits dangereux, et plus touchés par les accidents du travail : 10,8\% des hommes

6. Sauf précision, tous les chiffres cités sont issus de l'exploitation réalisée par la Dares de la dernière enquête Conditions de travail de 2013 (Algava, VincK, 2015 et 2016). 
déclarent avoir eu au moins un accident au cours des douze derniers mois, contre $7,1 \%$ des femmes.

Pour autant, les femmes ne sont pas épargnées par les pénibilités ou les risques professionnels. Le risque infectieux concerne 37,2\% d'entre elles, contre 28,6\% des hommes. Elles sont surexposées aux troubles musculosquelettiques (TMS) : elles représentent $58 \%$ des cas reconnus en 2003 (GuIGNON, 2008), cumulant un certain nombre de facteurs de risques de TMS dans leur travail. Ce dernier reste en effet plus répétitif $(44,9 \%$ des femmes déclarent que leur travail est répétitif, contre 37,5\% des hommes) et soumis à pression : 19,4\% des femmes indiquent devoir toujours se dépêcher dans leur travail, contre $15,5 \%$ des hommes. On observe aussi que $35 \%$ d'entre elles affirment ne pas pouvoir interrompre leur travail momentanément, contre $25,5 \%$ des hommes. Leurs supérieurs hiérarchiques leur disent plus souvent qu'aux hommes comment faire leur travail ; elles doivent aussi davantage que les hommes appliquer strictement des consignes. La plupart des femmes doivent faire un travail monotone, répétitif, sans possibilité de l'interrompre et de le faire varier (41,6\% des hommes peuvent faire varier les délais fixés dans leur travail, contre seulement 27,4\% des femmes).

Nombreux sont ainsi les signes de la faible autonomie des femmes par rapport à la définition de leur travail et d'une plus grande soumission des travailleuses à l'ordre organisationnel. Comme le montre Selma AMIRA (2010) à partir de l'exploitation d'une autre enquête, celle sur les changements organisationnels et l'informatisation de 20062007 (COI), les femmes occupent des emplois où le travail semble moins épanouissant (moins d'autonomie, moins de possibilités d'apprendre des choses nouvelles, etc.). Elles sont plus fréquemment confrontées aux pénibilités mentales : $52 \%$ d'entre elles travaillent en contact avec des personnes en situation de détresse, contre 36,9\% des hommes, et elles doivent plus souvent faire face à des agressions physiques ou verbales ou encore cacher leurs émotions au travail (seules $25 \%$ des femmes n'ont jamais à cacher leurs émotions au travail, contre 37,5\% des hommes).

Ces différences de pénibilités sont d'abord le reflet de la ségrégation professionnelle selon le sexe sur le marché du travail : si femmes et hommes ne sont pas soumis aux mêmes pénibilités et risques, c'est avant tout parce qu'elles et ils ne travaillent pas dans les mêmes professions. Les femmes actives sont nettement concentrées parmi les employés et les professions intermédiaires, et exercent majoritairement au contact du public (78,4\% des femmes en 2013), par exemple dans les soins médicaux et paramédicaux ou la prise en charge des enfants. En 2011, 97,7 \% des aides à domicile et des assistant.e.s maternel.le.s sont des femmes, tout comme $90,4 \%$ des aides-soignant.e.s, $87,7 \%$ des infirmier.ère.s et $66 \%$ des enseignant.e.s. Quant aux hommes, ils sont plus souvent employés dans des tâches techniques et de direction. Ils représentent $98 \%$ des ouvrier.ère.s qualifié.e.s du bâtiment, $92,1 \%$ des technicien.ne.s et agent.e.s de maîtrise du bâtiment et des travaux publics ou encore 79,7 \% des ingénieur.e.s de l'informatique (ARGOUARC'H, CALAVREZO, 2013). 


\section{Le genre des données en santé au travail}

\section{Faire émerger le genre des statistiques}

Certaines approches statistiques tentent de dépasser le constat de cette ségrégation en essayant de différencier par des régressions les effets de sexe et de profession (BUÉ, 2004 ; Gollac, Volkoff, 2006 ; BoufFartigue et al., 2010). Il s'agit de parvenir à distinguer ce qui, parmi les conditions de travail des femmes, est lié au type de profession qu'elles occupent, de ce qui est au contraire lié spécifiquement au fait d'être une femme « toutes choses égales par ailleurs ». Cela conduit parfois à inverser les constats établis sans prise en compte des effets de structure, c'est-à-dire « toutes choses inégales réunies » pour reprendre l'expression de François HérAN (1996, p. 14). Ainsi, en 2002, « une proportion de femmes nettement plus élevée que d'hommes (69\% et $56 \%$ ) déclare être en contact avec le public, alors que selon le modèle logit, à métier, âge et secteur identiques, la probabilité pour une femme d'être en contact avec le public est inférieure de $16 \%$ à celle d'un homme. [...] Le paysage des conditions de travail des femmes résulte largement des caractéristiques des professions dans lesquelles elles sont concentrées » (BUÉ, 2004, p. 80). Néanmoins cette distinction entre effet de profession et effet de sexe demeure ambiguë. L'effet de profession n'est lui-même pas indépendant du genre : les études sur la féminisation d'une profession ont montré de longue date les effets de dévalorisation que ce phénomène induisait (PERROT, 1987), notamment par le biais de mécanismes complexes de différenciations sexuées et de petites hiérarchies qui se rejouent ensuite à l'intérieur de chaque profession (Le Feuvre, Walters, 1993 ; Boigeol, 1993 ; Cacouault-Bitaud, 2001). Ce qu'est une profession à un moment donné, ses caractéristiques de travail, mais aussi le niveau des salaires, la reconnaissance des qualifications ou encore le prestige social associé - autant d'éléments importants pour la santé des salariés qui les occupent-, dépendent de son degré de féminisation ou de masculinisation (SILVERA, 2014).

L'ergonome Karen Messing (encadré) et les nombreux chercheurs et chercheuses qui travaillent avec elle sur ces questions ont depuis longtemps soulevé les multiples problèmes que posent les approches statistiques de la santé au travail. Non seulement femmes et hommes n'ont pas les mêmes professions, non seulement dans une même catégorie socioprofessionnelle (comme les ouvriers) ils n'ont pas les mêmes postes (ne sont pas dans les mêmes secteurs, les mêmes ateliers, etc.) mais dans un même poste, femmes et hommes ne font pas les mêmes tâches. "Par exemple, des observations effectuées au Québec ont montré que les hommes qui font le nettoyage en milieu hospitalier passent deux fois plus de temps que les femmes à nettoyer les planchers, mais la moitié moins à nettoyer les cuves de toilettes, avec des conséquences pour les postures et donc les sites corporels de troubles musculosquelettiques » (MESSING, 2014, p. 30). Une telle vigilance aux effets de genre conduit à mettre en évidence qu'il est difficile de prévoir les risques auxquels sont exposées les femmes préposées à l'entretien en hôpital à partir d'une étude ergonomique des caractéristiques du poste 
des hommes préposés à l'entretien... Or, cette dimension genrée n'est pratiquement jamais prise en compte dans l'expertise et la recherche.

\section{ENCADRÉ}

\section{Karen Messing (1943-) et l'ergonomie féministe au Canada}

Karen Messing est la figure de proue des approches féministes critiques des conditions de travail et de leurs conséquences sur la santé. Née aux États-Unis en 1943, elle étudie les sciences sociales à Harvard avant de se spécialiser dans la biologie, puis de poursuivre un doctorat en génétique. Elle s'installe à Montréal dans les années 1960, devient enseignante-chercheuse à l'université du Québec à Montréal (Uqam) en 1976 où elle collabore avec des syndicats et découvre la question des risques professionnels, à laquelle elle consacrera sa carrière. En 1990, K. Messing suit une formation en ergonomie au Conservatoire national des arts et métiers à Paris (Cnam), qui lui permet de confirmer la place importante des sciences sociales de terrain dans ses enquêtes, tout en gardant son ancrage dans les sciences naturelles. Elle revendique haut et fort une approche féministe critique des questions de santé au travail, qu'elle expose en particulier dans son livre One-Eyed Science: Occupational Health and Women Workers (Messing, 1998) ${ }^{1}$. « J'ai cherché à comprendre où la nature prend fin et où commence la culture », explique-t-elle (Messing, 2000, pp. 17-18). On peut parler d'une véritable école de travaux dans la suite de ceux de K. Messing, la plupart adossés institutionnellement au Centre de recherche sur les interactions biologiques entre la santé et l'environnement (Cinbiose) créé en 1985, aujourd'hui Centre de recherche interdisciplinaire sur le bien-être, la santé, la société et l'environnement, qui fait indiscutablement du Québec le principal foyer de réflexion pour une approche féministe des conditions de travail.

1. Le livre est traduit en France sous le titre La Santé des travailleuses. La science est-elle aveugle ? (MESSING, 2000). Mais on remarquera que dans la traduction française, l'idée portée par « one-eyed science » est reléguée en sous-titre et modifiée : la science borgne devient aveugle. On perd ainsi la subtilité de l'argument féministe selon lequel la science fermerait un œil aux dangers auxquels sont exposées les travailleuses, tout en gardant l'autre ouvert pour les risques professionnels des hommes.

Allant plus loin dans leur critique des approches statistiques, K. Messing et ses collègues montrent qu'une même variable des enquêtes sur les conditions de travail peut cacher des réalités bien différentes pour les femmes et les hommes. C'est le cas par exemple de la « posture statique debout ». À partir de l'étude du Quebec Health Social Survey (QHSS, une enquête basée sur un échantillon de 30000 individus) de 1998, ces chercheuses établissent que les hommes soumis habituellement à la posture statique debout au cours de leur journée de travail ont plus souvent la possibilité de se déplacer ou encore de s'asseoir que les femmes assujetties à cette même contrainte 
(Tissot, Messing, Stock, 2005). Pour ces raisons, la posture statique debout a des effets très différents sur la santé des femmes et des hommes, entraînant un risque accru pour les travailleuses de développer des TMS (Messing, LipPel, Stock, Tissot, 2011).

Enfin, K. Messing et son équipe plaident pour une approche statistique de la santé au travail qui soit dynamique, à l'échelle de carrières professionnelles. Cela permet par exemple de mieux comprendre la prévalence des TMS chez les femmes et la plus faible probabilité qu'ont les hommes de les développer : par exemple, «parmi les femmes dont le travail est répétitif, les travailleuses du vêtement et d'autres industries, les préposées à l'entretien, les coiffeuses et les secrétaires ou opératrices de saisie de texte souffrent de façon excessive de problèmes aux mains et/ou aux poignets, aux bras, aux épaules et au cou » (Messing, 2000, p. 127). Les enquêtes montrent que les hommes aussi effectuent des tâches répétitives. Cependant, ils obtiennent généralement des promotions - ce que Sophie Louey et Gabrielle Schütz appellent l'« escalator de verre » pour les hommes dans les métiers très féminisés, comme les fonctions d'accueil - qui, à un moment de leur carrière, les dégagent de ce genre de tâches (ANGELOFF, 2000 ; Louey, SchüTZ, 2014). Plusieurs études révèlent ainsi que les contraintes physiques diminuent avec l'âge pour les travailleurs, mais pas pour les travailleuses. L'évolution des carrières, même lorsqu'elle est peu flagrante car tassée au bas de l'échelle des emplois, a des effets sur les atteintes à la santé. Les formes d'emploi jouent également un rôle. Ainsi, les caissières à temps partiel travaillent plus souvent aux moments de très grande affluence, et rarement dans les périodes de creux. Elles ont donc plus de chances de développer des TMS que leurs collègues qui sont à temps plein (MESSING, 2000). De manière globale, comme le souligne Laurent VOGEL (2015) à partir de l'Enquête européenne sur les conditions de travail, le temps partiel est corrélé à une plus grande flexibilité des horaires, à moins de formation et à moins de chances de promotion, qui permettraient précisément d'échapper aux postes impliquant un travail très répétitif dans un cycle de temps court.

\section{Le travail des femmes moins dangereux que celui des hommes?}

L'un des apports majeurs de l'approche féministe est d'analyser de manière critique les processus genrés par lesquels certaines caractéristiques d'un travail sont constituées en tant que conditions de travail, et viennent à être considérées socialement et politiquement comme graves ou au contraire anodines. Cela vaut également pour l'examen des conséquences de ces conditions de travail dangereuses. En effet, les procédures de définition, de « reconnaissance » et d'indemnisation des maladies professionnelles et des accidents du travail varient fortement selon le genre.

Lorsque l'on rappelle que les femmes vivent plus longtemps que les hommes, on oublie qu'elles passent une plus grande partie de leur vie en mauvaise santé (RoBINE, Cambois, 2013). Les travaux menés au Cinbiose (encadré) critiquent l'idée selon laquelle les femmes seraient exposées à des conditions de travail moins dangereuses, et entraînant moins de conséquences néfastes sur leur santé, que leurs homologues 
masculins. Largement répandue, cette euphémisation par la comparaison peut conduire les chercheurs, les militants et les préventeurs à se désintéresser de l'identification et de la prévention des problèmes de santé spécifiques rencontrés par les travailleuses. La pénibilité des gestes répétitifs, de la station debout prolongée ou celle des postes particulièrement stressants - des conditions de travail qui incombent en priorité aux femmes - est souvent présentée comme moins grave que d'autres risques qualifiés de « lourds » (accidents, produits cancérogènes) que l'on rencontre dans des secteurs majoritairement masculins.

La question de la faible prise en compte des spécificités physiques ou psychiques des femmes au travail mérite également d'être posée. Certes, c'est un raisonnement risqué. Lorsqu'il est mobilisé, il aboutit en général à essentialiser certains traits en les considérant comme des désavantages proprement féminins, justifiant l'exclusion des femmes de pans entiers du marché du travail et leur cantonnement à des ghettos professionnels féminins. Les femmes seraient moins fortes physiquement, plus petites, plus fragiles, plus minutieuses, ou encore leur rôle dans la reproduction humaine entraînerait certains dangers spécifiques... Forte de son double ancrage en biologie et en sciences sociales, $\mathrm{K}$. Messing adopte une position subtile dans ce débat. Elle considère qu'il ne faut pas nier les différences moyennes de constitution physique qui existent entre femmes et hommes, tout en restant extrêmement vigilante pour éviter que ce constat ne soit utilisé dans un but d'exclusion des femmes. Elle a participé par exemple à une étude sur les femmes qui travaillent en chambre froide dans les abattoirs français, démontrant que l'irrégularité des horaires et l'exposition au froid avaient des effets sur la régularité du cycle menstruel (Messing, SAUREL-CubizolLEs, Bourgine, KAMINSKI, 1992). Cependant, dans un autre texte, les chercheuses remarquent :

«Le froid, l'intensité du travail et les horaires irréguliers affectent la physiologie de tous les travailleurs, et non uniquement celle des femmes. Est-ce une bonne idée de se focaliser sur leurs effets sur la santé menstruelle ? C'est en vérité une question de stratégie, qui doit être résolue par les travailleuses concernées. Nous-mêmes n'avons jamais eu connaissance d'un conflit de travail portant sur la santé menstruelle, et avons eu des difficultés à évoquer le sujet dans des discussions, y compris avec des syndicats majoritairement féminins ${ }^{7}$.»

(Messing, LipPel, Demers, Mergler, 2000, pp. 38-39)

Ce sont non seulement les risques au travail, mais aussi leurs conséquences sur la santé, qui bénéficient d'une visibilité différentielle selon le genre. Les institutions de prévention mettent en effet l'accent sur les accidents physiques ponctuels et dont les conséquences sont manifestes, qui concernent d'abord les hommes, plutôt que sur la mise en visibilité des lésions et des maladies chroniques d'origine professionnelle, qui affectent d'abord les femmes. Par ailleurs, les dispositifs de reconnaissance des maladies professionnelles comportent un certain nombre de « filtres » qui, à chaque étape, défavorisent la reconnaissance des pathologies contractées par les travailleuses

7. Nous traduisons. 
(WebB, et al., 1989). Au Canada, les travaux menés par la juriste Katherine Lippel montrent ainsi que le système d'indemnisation sous-reconnaît largement l'origine professionnelle des pathologies des travailleuses, au regard de ce qui est pratiqué pour les hommes. La chercheuse évoque une forme de « discrimination systémique ». Dans une enquête sur les recours en vue de la reconnaissance de l'origine professionnelle de TMS, un tirage aléatoire parmi les décisions de la commission de reconnaissance révèle que $36 \%$ des demandes féminines sont acceptées, contre $61 \%$ des demandes masculines (LIPPEL, 2003). Les problèmes de santé des femmes sont bien plus souvent renvoyés à des caractéristiques physiques ou psychiques « naturelles » préexistantes (LIPPEL, 2008). On retrouve une discrimination systémique similaire pour la reconnaissance des TMS en Suisse (Probst, 2009), des maladies professionnelles en Belgique (Vogel, 2011), des cancers professionnels en France (PAIVA, 2012, 2014), et en particulier des cancers de l'amiante (ChAmming's et al., 2013). En France toujours, une exploitation de l'enquête SIP attentive aux effets de genre montre que les femmes ont une plus faible probabilité que les hommes de voir reconnaître institutionnellement les accidents du travail ou les maladies professionnelles les concernant, à tel point que les auteurs concluent à un « handicap relatif des femmes dans la prise en charge » institutionnelle des atteintes à la santé liées au travail (Amossé, DAuBAs-LetourneuX, 2012, p. 65).

Toutes ces interrogations autour des différences genrées de traitement et de mise en visibilité des pénibilités, des risques, des expositions et des maladies professionnelles, s'accompagnent d'une critique et de propositions en termes de prévention (LEGRAND, 2015 ; CHAPPERT, THÉRY, 2016). De manière générale, il semble en effet presque toujours possible de convertir la question de l'adaptation du corps féminin à certains travaux en une autre : celle de l'adaptation du poste de travail aux spécificités du corps féminin moyen, voire du corps humain sans distinction de sexe (en demandant la suppression des risques qui valent pour tous).

«L'environnement de travail s'adapte aux caractéristiques physiques de la population pour laquelle il a été conçu. Les établis ont une certaine hauteur, les sacs de ciment ont un certain poids, les distances ont une certaine longueur, parce qu'un jugement a été explicitement ou implicitement porté au sujet des caractéristiques physiques moyennes de la main-d'œuvre. ${ }^{8}$ »

(Messing, LipPel, Demers, Mergler, 2000, pp. 25-26)

La perspective féministe en santé au travail invite à ne pas se centrer sur la seule question du libre accès des femmes aux différentes professions, mais également à demander que les outils, les équipements et les pratiques de travail soient adaptés afin de mieux convenir au corps féminin moyen. C'est ainsi que, dans une étude se déroulant dans une municipalité qui a commencé à recruter des femmes chez les jardiniers, les journaliers et les travailleurs du nettoyage, K. Messing souligne que « comme les hommes les plus petits, des femmes cols-bleus se trouv[ai]ent désavantagées par des outils trop grands pour leur main, des bottes trop grosses, un volant

8. Nous traduisons. 
trop loin du siège d'un chariot » (Messing, 1999, p. 4). Cette approche s'oppose à la position naturalisante dominante, décrite et critiquée par Louise Tilly et Joan Scott, qui a légitimé les employeurs dans leur refus d'adapter le travail aux contraintes spécifiques rencontrées par les femmes : «Si les femmes étaient par nature inaptes au travail industriel, pourquoi chercher à modifier les processus de travail, l'aménagement social de la maternité et du soin des enfants? »(TilLy, ScotT, 1987, p. 22).

\section{Explorer l'articulation entre travail domestique et travail rémunéré}

La citation qui précède nous invite à élargir notre champ d'étude. Pour importants qu'ils soient, les travaux présentés jusqu'ici ont pour limite de rester majoritairement centrés sur la sphère du travail rémunéré. Or, les femmes en couple accomplissent toujours deux tiers des tâches domestiques (Champagne et al., 2015). Comme le montre une longue tradition de recherches féministes qui ont pour l'essentiel porté sur des situations d'indépendance professionnelle et des entreprises familiales, elles jouent également un rôle majeur dans le soutien à la carrière de leur conjoint (BARTHEZ, 1982 ; SCHWEITZER, 2002 ; BessiÈre, GolLAC, 2014). Les études sur la santé au travail gagnent à importer ces perspectives, tout à fait pertinentes y compris dans le cadre de ménages dont les membres ont des activités salariées.

\section{«Famille-travail : une perspective radicale ${ }^{9}$ »}

S'il est d'usage de « constater » que les femmes travaillent moins que les hommes, cela n'est plus vrai dès lors qu'on prend en compte le travail non rémunéré, comme le suggère L. Vogel à partir des données européennes sur les conditions de travail, ainsi que des données nationales venant d'Espagne et d'Italie : « Si l'on additionne le travail rémunéré, le temps de transport et le travail non rémunéré, le temps de travail hebdomadaire moyen des femmes s'élève à 64 heures contre 53,4 heures pour les hommes » en Europe (Vogel, 2015, p. 13). Une telle approche permet aussi de reconsidérer les données sur les accidents du travail. Les accidents causés par le travail non rémunéré sont très mal documentés en France, comme plus largement en Europe. Néanmoins l'Italie recueille des données plus systématiques dans la mesure où elle a mis en place un système d'indemnisation des accidents du travail domestique par un décret adopté en septembre 2000. D'après les recherches portant sur l'année 2007 citées par L. Vogel, environ 400000 femmes se seraient présentées aux services de premier secours des hôpitaux italiens à la suite d'accidents survenus à la maison - c'est à peu près le nombre d'accidents du travail comptabilisés pour les femmes sur une

9. Nous reprenons ici le titre du numéro spécial consacré par la revue Nouvelles Questions féministes (BACHMANN et al., 2004) qui remet à l'honneur la perspective des années 1970 articulant travail rémunéré et travail domestique. 
année en France d'après l'enquête Conditions de travail de 2013 -, et 110000 de ces accidents seraient causés par le travail domestique. Une hospitalisation s'est imposée dans 9200 cas et des invalidités graves permanentes concerneraient chaque année environ 900 femmes.

Étudier conjointement le «travail professionnel » et le «travail domestique » - pour reprendre une figure de style mobilisée dans les années 1980 (DAUNE-RICHARD, 1983) - est difficile à traduire en dispositif de recherche du fait de la disjonction de lieux. Il est cependant possible d'entrer dans ces deux mondes au moins indirectement, en menant des entretiens ouverts et, lorsque c'est possible, en présence des différents membres du ménage. Dans un article devenu un classique de la sociologie, Michel Pialoux explique comment, alors qu'il effectue un entretien à domicile avec un chef d'équipe nommé Étienne T., dans le cadre de la post-enquête de la Dares sur les conditions de travail de 1991, il en vient à réaliser un double entretien sur le travail ouvrier (PialouX, 1995). La femme d'Étienne, Josiane T., ouvrière elle-même, qui écoute l'air de rien depuis la pièce à côté où elle refait le papier peint, finit par donner son point de vue. M. Pialoux montre que cette situation d'entretien en famille ne conduit pas seulement à deux points de vue sur le travail ouvrier qui ne feraient que s'additionner : à de multiples moments, Josiane, en révélant les conséquences du travail à l'usine sur leur intimité, dévoile certains traits des pénibilités du travail d'Étienne que lui-même, en tant que chef d'équipe, ne peut voir ou dire. Le sociologue suggère ainsi la richesse en propre d'une étude des conditions de travail à partir de la sphère familiale.

Le dossier que nous proposons sous le titre «Quand la pénibilité du travail s'invite à la maison » a pour ambition de continuer à creuser le sillon d'une perspective féministe sur la santé au travail en réinvestissant l'articulation entre sphère du travail et sphère de la famille. En effet, déjà à la fin des années 1970 et au début des années 1980, la sociologie féministe francophone avait renouvelé l'étude du travail en proposant d'analyser conjointement la sphère du travail et la sphère de la famille, les interactions entre travail productif et travail reproductif (BATTAGLIOLA, 1984 ; COLLECTIF, 1984), refusant d'adopter la catégorie politique et euphémisante de « conciliation » travailfamille (BACHMANN et al., 2004). D'un côté, ce qui se passait dans la famille, comme l'inégal partage du travail domestique, la nature des tâches revenant à chacun des conjoints ou encore le type d'organisation domestique et les rapports de pouvoir, a été éclairé sous un nouveau jour par l'étude des formes d'emploi, de carrière et de place dans la division du travail des membres du couple (SingLY, 1987 ; COMMAILle, 1993). De l'autre, des thèmes classiquement étudiés à travers des caractéristiques du travail comme les carrières professionnelles, la répartition des postes et des tâches, les niveaux de qualification, la place dans la hiérarchie, les manières de faire son travail ou encore le rapport entretenu à l'emploi et au travail, ont ainsi été rendus intelligibles par l'observation de ce qui se passait dans la famille (BerThauX-WiAME, 1982 ; DAUnERICHARD, 1983 ; BARTHEZ, 1984).

Alors que cette approche continue à faire ses preuves dans les travaux qui s'intéressent aux inégalités de genre dans les univers professionnels (GADÉA, MARRY, 2000 ; 
Guillaume, Pochic, 2007), elle n'a guère été investie par les spécialistes de la santé au travail. On peut notamment faire l'hypothèse que, du fait de leur mode d'approche et d'action - observer les postes de travail et discuter avec les salariés in situ pour modifier les conditions de travail -, l'ergonomie comme la psychodynamique du travail n'ont pas pour priorité d'intégrer la vie familiale dans l'analyse des problèmes de santé au travail. La préoccupation est pourtant présente dans certains travaux, comme ceux sur les travailleurs sous-traitants du nucléaire (THÉBAUD-MonY, 2000), et certaines enquêtes statistiques récentes sur la santé au travail, comme SIP, incluent désormais des questions à la fois sur la vie familiale et sur les parcours personnels. Les bases de données de ces enquêtes demeurent toutefois sous-utilisées dans cette perspective.

\section{Parcours de lecture : des résultats transversaux qui en appellent à de nouvelles recherches}

Ce numéro de la revue Travail et Emploi repose sur quatre contributions qui toutes prennent appui sur des enquêtes de terrain approfondies auprès de travailleurs, de travailleuses et de leur famille, le plus souvent leur conjoint ou conjointe, mais aussi quelquefois leurs parents et leurs enfants, enquêtes qui se situent dans des secteurs et des régions variés en France : la pêche en Vendée et en Bretagne, la maintenance de différents sites nucléaires sur tout le territoire, la recherche dans une grande entreprise énergétique en Île-de-France, une association de victimes des pesticides regroupant des agriculteurs de l'Ouest et du Sud-Ouest pour l'essentiel. Nous souhaitons insister ici sur quatre résultats communs mis en lumière par les contributions de ce dossier et qui constituent autant de pistes pour des recherches à venir.

Tout comme le nuage de Tchernobyl ne s'est pas arrêté aux frontières de la France, les risques et les pénibilités liés à la sphère professionnelle ne s'arrêtent pas aux portes des foyers. Tel est le premier résultat mis en évidence par l'ensemble des articles. Les voies par lesquelles ces risques et pénibilités du travail pénètrent la sphère de la famille sont multiformes. Ce sont d'abord les contraintes du temps de travail qui s'invitent dans les foyers. Dans leur article sur les cadres occupant des positions de chercheurs dans une grande entreprise de l'énergie, Lucie Goussard et Guillaume Tiffon s'intéressent aux débordements du travail dans la famille, à ce qu'ils appellent le « surtravail » (le travail effectué en dehors du travail, c'est-à-dire à la maison). Celui-ci occupe des espaces inattendus et inégalement distribués selon le sexe des travailleurs concernés : si tel directeur de département peut demander à sa femme de lui laisser la maison le week-end (elle emmène les enfants chez les grands-parents), telle chercheuse doit s'isoler la nuit pour travailler dans les toilettes. Les hommes occupent les espaces domestiques pour y travailler, tandis que les femmes doivent bricoler avec le temps et l'espace domestiques pour y grappiller des moments de travail (CHABAUD-RYCHTER et al., 1985).

Les relations familiales sont également particulièrement touchées par les risques encourus dans ces situations de surtravail, notamment les facteurs de risques 
psychosociaux et leurs conséquences (irritabilité, insomnies, dépression, etc.). Une cadre se compare ainsi à un pitbull à la maison pour résumer l'effet du surtravail sur sa vie privée et les relations avec ses enfants. Paradoxalement, Marie Charvet, Fabienne Laurioux et Gilles Lazuech nous montrent que c'est aussi par l'absence des travailleurs que la pénibilité du travail peut retentir sur la famille. Les pêcheurs qui partent de longs mois en mer laissent leur épouse assumer l'ensemble des contraintes et des responsabilités domestiques. Les caractéristiques du travail des conjoints retentissent sur la charge physique, mais aussi sur la charge mentale, du membre du ménage qui exécute le travail domestique (HAICAULT, 1984). Une telle analyse invite à étudier dans le détail les variations du travail domestique en relation avec celles des conditions du travail rémunéré.

Par-delà la manière dont les rythmes du travail s'imposent aux familles, et différentiellement aux femmes et aux hommes, les formes d'exposition de la famille aux risques du travail sont parfois très directes, et pourtant complètement déniées encore aujourd'hui. Les conjointes et les enfants des agriculteurs victimes des pesticides peuvent difficilement avoir été eux-mêmes épargnés par les épandages réalisés sur l'exploitation familiale. Selon Giovanni Prete et Jean-Noël Jouzel, les femmes d'agriculteurs ont d'autant plus de mal à évoquer les problèmes de santé dus aux pesticides, auxquels elles-mêmes et leur conjoint doivent faire face, que ces derniers ont une responsabilité implicite dans la contamination de leurs proches. Comme le montre Marie Ghis Malfilatre, les travailleurs du nucléaire subissent des contaminations qui sont censées rester à l'intérieur de la centrale. Pourtant, un matin, Louis ne peut entrer dans la centrale car il dépasse les niveaux de contamination tolérés. Il comprend alors que les sous-vêtements qu'il ramène le soir à la maison, et que sa conjointe lave avec le reste du linge de la famille, sont parfois fortement chargés de poussières radioactives. Amalia et Louis découvrent ainsi tout à fait par hasard à quel point les radiations ont pénétré leur intimité.

Un autre résultat mis en lumière par les contributions de ce dossier est l'importance du travail domestique invisible des femmes pour gérer les risques rencontrés par leur conjoint dans l'exercice de son métier. Le regard sociologique gagne ici à distinguer un double effet de position et de socialisation (AvrIL, CARTIER, SERRE, 2010, pp. 121181). La position des femmes dans les rapports sociaux de sexe, et notamment leur assignation à la sphère domestique, les conduit à porter un regard spécifique sur le travail, et en particulier sur celui de leur conjoint. Danièle KeRGOAT (1978) avait suggéré que les femmes ont un regard plus distant à l'égard de la sphère professionnelle, du fait qu'il ne s'agit pour elles que d'une préoccupation parmi d'autres. Lorsque les femmes ont un emploi, elles occupent plus souvent des positions hiérarchiques inférieures à celles de leur mari, ce qui les porte, comme dans l'entretien réalisé par Michel Pialoux, à avoir un regard critique, ou tout au moins décalé, sur la situation de travail de leur conjoint. De plus, tout un courant de la sociologie des mouvements sociaux adoptant une perspective féministe a montré que les femmes, parce qu'elles ont plus souvent la charge des enfants, sont plus susceptibles de se mobiliser face aux 
risques de pollution environnementale, comme l'exposition à des produits toxiques dans des situations de la vie quotidienne (Krauss, 1993 ; Brown, Ferguson, 1995).

Dans la lignée de ces travaux qui interrogent les effets spécifiques de la position des femmes - dans les rapports sociaux de sexe et dans le monde du travail - sur leur rapport critique au travail et à ses risques, les contributions du dossier montrent que ce sont les femmes qui la plupart du temps alertent leur conjoint et tentent de les mobiliser pour qu'ils fassent reconnaître les atteintes à la santé qu'ils ont subies au travail. Ainsi, les femmes d'agriculteurs de l'association Phyto-victimes, tout comme les conjointes des travailleurs du nucléaire, ont souvent joué un rôle déterminant pour que leur conjoint entame une procédure de reconnaissance de maladie professionnelle.

Par-delà ce premier niveau d'analyse en termes de position, le dossier permet d'approfondir le rôle spécifique joué par les femmes du fait de leurs dispositions acquises sur différentes scènes socialisatrices tout au long de leur trajectoire. Si leurs comportements tiennent pour partie à leur position relative dans des rapports inégaux, il est également éclairé par leur « condition », c'est-à-dire par leurs socialisations passées et présentes découlant de leurs trajectoires (BouRdiEu, 1966). On sait en effet que les femmes, notamment dans certaines fractions des milieux populaires, sont habituées parfois très jeunes (en particulier les filles d'immigrées) à « faire les papiers » de la famille (SiBLOT, 2006) et, ce faisant, développent des dispositions administratives qu'une situation de maladie professionnelle grave du conjoint peut les conduire à réactiver (MARCHAND, 2016). Les différentes contributions du dossier creusent cette piste tout en l'élargissant. La socialisation administrative des femmes de pêcheurs, d'agriculteurs ou de travailleurs du nucléaire - ce n'est certainement pas un hasard si l'on rencontre autant d'anciennes secrétaires parmi elles - éclaire leur mobilisation pour la reconnaissance des atteintes à la santé de leur mari. Pour le dire autrement, il semblerait qu'un travailleur a d'autant plus de chances de faire reconnaître sa maladie professionnelle que sa conjointe a accumulé au cours de sa trajectoire scolaire et/ou professionnelle des ressources administratives, mêmes modestes, ou a été proche du milieu de la santé (MARICHALAR, 2016). Une analyse des inégalités en termes de reconnaissance des atteintes à la santé gagnerait à étendre la perspective d'étude pour intégrer la trajectoire sociale des conjointes.

Alors que la plupart des travaux mettent l'accent, dans la continuité de leurs rôles domestiques, sur le soutien moral et affectif apporté par les femmes ou bien sur le réinvestissement de compétences domestiques, certaines contributions du dossier donnent également à voir un phénomène jusque-là peu mis en évidence : la mobilisation par les femmes de leur capital économique et de leur capital social. Certaines conjointes de travailleurs du nucléaire reprennent ainsi une activité professionnelle pour soutenir leur conjoint dans une démarche de reconnaissance de maladie professionnelle et font vivre la famille pendant toute la procédure. Les femmes de pêcheurs, habituées qu'elles sont à gérer les relations avec les banques, à négocier des emprunts, sont en première ligne lorsqu'il s'agit de permettre au ménage de continuer à vivre alors que l'état de santé de leur mari ne lui permet plus de travailler. Et ce n'est peut-être pas fortuit si les 
agriculteurs qui militent dans l'association Phyto-victimes ont souvent une conjointe salariée en dehors de l'exploitation. Par exemple, l'une d'elles mobilise une avocate rencontrée dans le cadre de son travail pour appuyer le dossier de son mari.

Les articles de ce numéro invitent encore à réfléchir aux différences de pratiques des femmes et des hommes, en tant que travailleuses et travailleurs, face aux risques encourus et aux démarches de reconnaissance. On ne peut qu'être frappé par le fait que trois des contributions sur quatre portent sur des cas de femmes se mobilisant dans l'ombre pour les problèmes de santé au travail de leur conjoint et qu' aucune n'évoque le cas inverse, en cohérence d'ailleurs avec ce que met en évidence la littérature dans ce domaine. Cela reflète bien évidemment la division sexuelle des pénibilités et des risques soulignée au début de cette introduction (les accidents du travail et les maladies professionnelles invalidantes ou conduisant rapidement à la mort étant plus fréquents dans les métiers où les hommes sont majoritaires). Mais cela relève aussi sûrement d'autres phénomènes, comme le déni des risques encourus par les femmes dans leurs métiers plus sédentaires, les difficultés qu'elles ont à s'envisager comme des travailleuses exposées, mais aussi celles des sociologues à les considérer comme telles.

On insistera enfin sur un dernier grand type de résultat mis en lumière par l'ensemble des articles de ce dossier : le caractère non mécanique des effets des pénibilités du travail dans la sphère familiale et notamment au sein des couples. Toutes les contributions montrent qu'il n'y a pas d'effet simple du genre et que seule l'étude localisée des facteurs en jeu permet d'éclairer les comportements (PAILlEt, SERRE, 2014). Si dans le nucléaire, les conjointes jouent parfois un rôle de soutien moral et matériel, il arrive aussi que les atteintes à la santé, ainsi que les " grands déplacements » imposés par l'activité de maintenance des centrales conduisent à des séparations, comme le souligne subtilement Marie Ghis Malfilatre. De même Marie Charvet, Fabienne Laurioux et Gilles Lazuech mettent en évidence la grande diversité des ordres négociés à l'intérieur des couples pour faire face aux conditions de travail dans la pêche : à côté des configurations dites de «pénibilité soutenue » où les conjointes de pêcheurs acceptent sans contrepartie de soutenir toutes les formes de pénibilités qui découlent de l'activité de leur mari, ils exposent ce qu'ils nomment la « pénibilité négociée ». Ils mettent ainsi en avant des formes de conjugalité où les pénibilités du travail sont plus explicitement discutées, au cœur de négociations au sein du couple, et peuvent donner lieu à des concessions réciproques. Lorsque ces négociations échouent, c'est aussi la rupture conjugale qui se profile et peut en grande partie être éclairée par le retentissement des pénibilités du travail dans la sphère familiale.

Ce dossier n'est qu'un premier pas dans un programme de recherche sur la santé des femmes et des hommes, qui mènerait conjointement l'étude de ce qui se joue dans le travail rémunéré et dans le travail domestique. Il serait souhaitable qu'à l'avenir, les protocoles de recherche mis en œuvre questionnent systématiquement cette dimension, se donnent véritablement les moyens de saisir ce qui se trame dans l'espace de la maison et de conduire des entretiens avec l'ensemble des membres des ménages, en partant du présupposé que chacun et chacune sont des travailleurs et des travailleuses 
à part entière, plutôt qu'uniquement des conjointes et des conjoints. Il n'en reste pas moins que, comme ce parcours de lecture et cette mise en perspective viennent de le suggérer, le dossier qui suit propose déjà de multiples pistes à explorer pour renouveler l'étude de la santé au travail des femmes et des hommes.

\section{BibliogRAPHIE}

Algava É., VinCK L. (2015), « Contraintes physiques, prévention des risques et accidents du travail. Enquêtes Conditions de travail », Synthèse.stat', n ${ }^{\circ} 10$.

Algava É., VincK L. (2015), « Intensité du travail et usages des technologies de l'information et de la communication. Enquêtes Conditions de travail », Synthèse.stat', nº 14.

Algava É., VincK L. (2015), «Autonomie dans le travail. Enquêtes Conditions de travail », Synthèse.stat', n ${ }^{\circ} 16$.

AlgaVA É., VincK L. (2016), « Les rapports sociaux au travail. Enquêtes Conditions de travail », Synthèse.stat', $\mathrm{n}^{\circ} 20$.

AlGAVA É., VINCK L. (2016), « Vécu du travail : reconnaissance, conflits de valeurs, insécurité et changements dans le travail. Enquêtes Conditions de travail », Synthèse.stat', n 21.

AmiRA S. (2010), « Les femmes occupent des emplois où le travail semble moins épanouissant », Dares analyses, $\mathrm{n}^{\circ} 082$.

Amossé T., Daubas-Letourneux V. (coord), Le Roy F., Meslin K., Barragan K., (2012), « Les accidents du travail et problèmes de santé liés au travail dans l'enquête SIP. (In)visibilités et inscription dans les trajectoires professionnelles », Rapport de recherche CEE, $\mathrm{n}^{\circ} 76$.

Angeloff T. (2000), Le Temps partiel : un marché de dupes ?, Paris, Syros.

ARgouarC'H J., CALAVRezo O. (2013), « La répartition des hommes et des femmes par métier : une baisse de la ségrégation depuis 30 ans », Dares analyses, $\mathrm{n}^{\circ} 79$.

Avril C., Cartier M., Serre D. (2010), Enquêter sur le travail. Concepts, méthodes, récits, Paris, La Découverte.

Bachmann L., Golay D., Messant F., Modak M., Palazzo C., Rosende M. (coord.) (2004), «Famille-Travail : une perspective radicale », Nouvelles Questions féministes, vol. 23, n 3 .

BARTHEZ A. (1982), Famille, travail et agriculture, Paris, Economica.

BARTHEZ A. (1984), «Femmes dans l'agriculture et travail familial », Sociologie du travail, vol. 26, n 3, pp. 255-267.

BATTAGLIOLA F. (1984), «Employés et employées : trajectoires professionnelles et familiales », in Collectif, Le Sexe du travail. Structures familiales et système productif, Grenoble, Presses universitaires de Grenoble, pp. 57-68.

BERCOT R. (dir.) (2014), La Santé au travail au prisme du genre : épistémologie, enquêtes et perspectives internationales, Toulouse, Octarès. 
BERCOT R. (dir.) (2015), Le Genre du mal-être au travail, Toulouse, Octarès.

BerthauX-WiAme I. (1982), «L'installation dans la boulangerie artisanale », Sociologie du travail, vol. 24, $\mathrm{n}^{\circ} 1$, pp. 8-23.

Bessière C., Gollac S. (2014), « Famille », in Chauvin P.-M., Grossetti M., Zalio P.-P. (dir.), Dictionnaire sociologique de l'entrepreneuriat, Paris, Presses de Sciences Po, pp. 274-287.

Boigeol A. (1993), « La magistrature française au féminin : entre spécificité et banalisation », Droit et société, $\mathrm{n}^{\circ} 25$, pp. 489-523.

Bouffartigue P., Pendariès J.-R., Bouteiller J. (2010), « La perception des liens travail/ santé. Le rôle des normes de genre et de profession », Revue française de sociologie, vol. 51, $\mathrm{n}^{\mathrm{o}} 2$, pp. 247-280.

Bourdieu P. (1966), «Condition de classe et position de classe », Archives européennes de sociologie, vol. 7, $\mathrm{n}^{\mathrm{o}} 2$, pp. 201-223.

Brown P., Ferguson F. I. T. (1995), “Making a Big Stink: Women's Work, Women’s Relationships, and Toxic Waste Activism”, Gender and Society, vol. 9, n 2, pp. 145-172.

BuÉ J. (2004), « Hommes et femmes face aux pénibilités du travail », in Bué J., Coutrot T., Puech I. (coord.), Conditions de travail, les enseignements de vingt ans d'enquêtes, Toulouse, Octarès, pp. 77-88.

Cacouault-Bitaud M. (2001), « La féminisation d'une profession est-elle le signe d'une baisse de prestige ?», Travail, genre et sociétés, $\mathrm{n}^{\circ} 5$, pp. 91-115.

Chabaud-Rychter D., Fougeyrollas-Schwebel D., Sonthonnax F. (1985), Espace et temps du travail domestique, Paris, Librairie des Méridiens.

Chamming's S., Clin B., Brochard P., Astoul P., Ducamp S., Galateau-Salle F., Ilg A. G., Goldberg M., Gramond C., Imbernon E., Rolland P., Pairon J.-C. (2013), “Compensation of Pleural Mesothelioma in France: Data from the French National Mesothelioma Surveillance Programme", American Journal of Industrial Medicine, vol. 56, n 2, pp. 146-154.

Champagne C., Pailhé A., Solaz A. (2015), « Le temps domestique et parental des hommes et des femmes : quels facteurs d'évolution en 25 ans ? », Économie et statistique, n ${ }^{\circ} 478-479-480$, pp. 209-238.

CHAPPert F., ThÉRY L. (2016) «Égalité entre les femmes et les hommes et santé au travail. Comment le genre transforme-t-il l'intervention sur les conditions de travail ? », Perspectives interdisciplinaires sur le travail et la santé, vol. 18, $\mathrm{n}^{\circ} 2$; en ligne : http://pistes.revues.org/4882, consulté le 9 mars 2016.

Collectif (1984), Le Sexe du travail. Structures familiales et système productif, Grenoble, Presses universitaires de Grenoble.

Commaille J. (1993), Les Stratégies des femmes. Travail, famille et politique, Paris, La Découverte.

Daubas-Letourneux V., ThéBaud-Mony A. (2001), « Les angles morts de la connaissance des accidents du travail », Travail et Emploi, n ${ }^{\circ} 88$, pp. 25-42. 
DAune-Richard A.-M. (1983), « Travail professionnel et travail domestique : le travail et ses représentations au sein des lignées féminines », Travail et Emploi, n 17, pp. 49-55.

FORTINO S. (2014), « Invisibilisation de la pénibilité au travail, mixité et rapports de genre. Conducteurs et agents commerciaux dans le secteur ferroviaire ", La Nouvelle Revue du travail, $\mathrm{n}^{\mathrm{o}} 4$; en ligne : http://nrt.revues.org/1422, consulté le 9 mars 2017.

FouQuet A. (2003), « La statistique saisie par le genre », in Laufer J., Marry C., Maruani M. (dir.), Le Travail du genre. Les sciences sociales du travail à l'épreuve des différences de sexe, Paris, La Découverte, pp. 280-298.

GADÉA C., MARRY C. (2000), « Les pères qui gagnent. Descendance et réussite professionnelle chez les ingénieurs », Travail, genre et sociétés, n ${ }^{\circ} 3$, pp. 109-135.

Gollac M. (1997), « Des chiffres insensés ? Pourquoi et comment on donne un sens aux données statistiques ? », Revue française de sociologie, vol. 38, nº 1, pp. 5-36.

Gollac M., Volkoff S. (2002), « La mise au travail des stéréotypes de genre. Les conditions de travail des ouvrières », Travail, genre et sociétés, ${ }^{\circ}$ 8, pp. 25-53.

Gollac M., Volkoff S. (2006), «La perception subjective du travail : rôle des identités de genre et des conditions d'emploi (quelques éléments d'analyse statistique) », Document de travail du CEE, n 69.

GousSARD L. (2014), « Organisation par projet et débordement du travail : des modalités genrées ? », in Bercot R. (dir.), La Santé au travail au prisme du genre : épistémologie, enquêtes et perspectives internationales, Toulouse, Octarès, pp. 79-98.

Guignon N. (2008), « Risques professionnels : les femmes sont-elles à l'abri ? », in Institut national de la statistique et des études économiques (Insee), Femmes et hommes. Regards sur la parité, Paris, Insee, pp. 51-63.

Guillaume C., Pochic S. (2007), « La fabrication organisationnelle des dirigeants. Un regard sur le plafond de verre », Travail, genre et sociétés, n 17, pp. 79-103.

Haicault M. (1984), « La gestion ordinaire de la vie en deux », Sociologie du travail, vol. 26, $\mathrm{n}^{\mathrm{o}} 3$, pp. 268-277.

HATZFELD N. (2008), «Affections périarticulaires : une longue marche vers la reconnaissance (1919-1991) », Revue française des affaires sociales, $\mathrm{n}^{\circ} 2-3, \mathrm{pp} .141-160$.

HÉrAn F. (1996), « L'école, les jeunes et les parents. Approches à partir de l'enquête Éducation. Présentation générale », Économie et statistique, n 293, pp. 5-15.

Huet M. (1983), « La concentration des emplois féminins », Économie et statistique, $\mathrm{n}^{\circ} 154$, pp. 33-46.

KeRgOAT D. (1978), « Ouvriers = ouvrières ? Propositions pour une articulation théorique de deux variables : sexe et classe sociale », Critique de l'économie politique, Nouvelle série, $\mathrm{n}^{\circ} 5$, pp. 65-97 (réédité in Kergoat D. [2012], Se battre disent-elles..., Paris, La Dispute).

Krauss C. (1993), "Women and Toxic Waste Protests: Race, Class and Gender as Resources of Resistance", Qualitative Sociology, vol. 16, n 3, pp. 247-262. 
LAGRAVE R.-M. (1990), « Recherches féministes ou recherches sur les femmes ? », Actes de la recherche en sciences sociales, $\mathrm{n}^{\circ} 83$, pp. 27-39.

LEGRAND É. (2015), Santé reproductive et travail : la prévention des risques reprotoxiques, rapport final de projet dans le cadre du programme national de recherche Environnement-santétravail, Maisons-Alfort, Anses ; en ligne : https://www.anses.fr/fr/system/files/ANSES-APRSHS-Sante\%20reproductive\%20et\%20travail-2015.pdf, consulté le 9 mars 2017.

Le Feuvre N. (2014), «Quelques défis pour l'appréhension sociologique des souffrances au travail sous l'angle du genre », in Bercot R. (dir.) (2014), La Santé au travail au prisme du genre : épistémologie, enquêtes et perspectives internationales, Toulouse, Octarès, pp. 105-125.

Le Feuvre N., Walters P. (1993), «Égales en droit? La féminisation des professions juridiques en France et en Grande-Bretagne », Sociétés contemporaines, n ${ }^{\circ}$ 16, pp. 41-62.

LENOIR R. (1980), « La notion d'accident du travail : un enjeu de luttes », Actes de la recherche en sciences sociales, $\mathrm{n}^{\circ} 32-33$, pp. 77-88.

LiPPEL K. (2003), “Compensation for Musculoskeletal Disorders in Quebec: Systemic Discrimination against Women Workers?”, International Journal of Health Services, vol. 33, $\mathrm{n}^{\mathrm{o}} 2$, pp. 253-281.

LiPPEL K. (2008), “Workers' Compensation and Controversial Illnesses”, in Moss P., Teghtsoonian K., Contesting Illness: Process and Practices, Toronto, University of Toronto Press, pp. 47-68.

Louey S., SchüTz G. (2014), « Les effets de la mixité au prisme du corps et de la sexualité : les hommes dans les métiers d'accueil », Travail et Emploi, nº 140, pp. 5-19.

Marchand A. (2016), «Quand les cancers du travail échappent à la reconnaissance. Les facteurs du non-recours au droit », Sociétés contemporaines, $\mathrm{n}^{\mathrm{o}}$ 102, pp. 103-128.

Marichalar P. (2016), « “C'est gênant de se mettre à dos son médecin, parce qu'on en a besoin." Ouvriers malades de leur travail face à la médecine », Agone, n 58, pp. 105-123.

Messing K. (1998), One-Eyed Science: Occupational Health and Women Workers, Philadelphia, Temple University Press.

Messing K. (1999), «La pertinence de tenir compte du sexe des "opérateurs” dans les études ergonomiques : bilan de recherches », Perspectives interdisciplinaires sur le travail et la santé, vol. $1, \mathrm{n}^{\circ} 1$; en ligne : https://pistes.revues.org/3840, consulté le 9 mars 2017.

MESsING K. (2000), La Santé des travailleuses. La science est-elle aveugle ?, Montréal, Éditions du Remue-ménage ; Toulouse, Octarès.

Messing K. (2014), « Genre, sexe, bien-être, conditions de travail et interface travail/famille dans l'enquête Eqcotesst », in Bercot R. (dir.), La Santé au travail au prisme du genre : épistémologie, enquêtes et perspectives internationales, Toulouse, Octarès, pp. 25-48.

Messing K., Lippel K., Demers D., Mergler D. (2000), "Equality and Difference in the Workplace: Physical Job Demands, Occupational Illnesses, and Sex Differences", NWSA Journal, vol. 12, n 3 , pp. 21-49. 
Messing K., Lippel K., Stock S., Tissot F. (2011), « Si le bruit rend sourd, rend-il nécessairement sourde ? Le défi d'appliquer l'analyse différenciée selon le sexe à la recherche d'informations sur la santé et la sécurité du travail », Revue multidisciplinaire sur l'emploi, le syndicalisme et le travail, vol. 6, n ${ }^{\circ}$ 2, pp. 3-25.

Messing K., Saurel-Cubizolles M.-J., Bourgine M., Kaminski M. (1992), “Menstrual-Cycle Characteristics and Work Conditions of Workers in Poultry Slaughterhouses and Canneries", Scandinavian Journal of Work, Environment \& Health, vol. 18, n 5, pp. 302-309.

Molinié A.-F., Volkoff S. (1980), « Les conditions de travail des ouvriers... et des ouvrières », Économie et statistique, $\mathrm{n}^{\circ} 118$, pp. 25-39.

PAillet A., SERRE D. (2014), « Les rouages du genre. La différenciation des pratiques de travail chez les juges des enfants », Sociologie du travail, vol. 56, n 3, pp. 342-364.

Paiva M. (2012), « Des femmes invisibles », Plein droit, nº 93, pp. 21-24.

PAIVA M. (2014), « Le sexe du cancer professionnel : le strictement féminin et l'inévitablement apolitique », Raison présente, $\mathrm{n}^{\circ} 190$, pp. 55-67.

Perrot M. (1987), «Qu'est-ce qu'un métier de femmes ?», Le Mouvement social, $\mathrm{n}^{\circ}$ 140, pp. 3-8.

Pfefferkorn R., Polesi H. (dir.) (2014), « Genre et santé au travail », Raison présente, $\mathrm{n}^{\circ} 190$.

Pialoux M. (1995), «L'ouvrière et le chef d'équipe ou comment parler du travail ? », Travail et Emploi, $\mathrm{n}^{\circ}$ 62, pp. 4-39.

PRoBSt I. (2009), « La dimension de genre dans la reconnaissance des TMS comme maladies professionnelles », Perspectives interdisciplinaires sur le travail et la santé, vol. 11, $\mathrm{n}^{\circ} 2$; en ligne : https://pistes.revues.org/2395, consulté le 9 mars 2017.

RAMAZZINI B. (1777), Essai sur les maladies des artisans, Paris, Moutard (la version originale en latin a été publiée en 1700).

Robine J.-M., CAmbois E. (2013), « Les espérances de vie en bonne santé des Européens », Population et sociétés, ${ }^{\circ} 499$.

Rosental P.-A., Devinck J.-C. (2007), « Statistique et mort industrielle. La fabrication du nombre de victimes de la silicose dans les houillères en France de 1946 à nos jours », Vingtième siècle. Revue d'histoire, $\mathrm{n}^{\circ}$ 95, pp. 75-91.

SCHWEITZER S. (2002), Les Femmes ont toujours travaillé. Une histoire de leurs métiers, XIX et $X X^{e}$ siècle, Paris, Odile Jacob.

SiBLOT Y. (2006), « "Je suis la secrétaire de la famille !" La prise en charge des tâches administratives entre subordination et ressource », Genèses, n 64, pp. 46-66.

SiLVERA R. (2014), Un Quart en moins. Des femmes se battent pour en finir avec les inégalités de salaire, Paris, La Découverte.

Singly (DE) F. (1987), Fortune et infortune de la femme mariée. Sociologie de la vie conjugale, Paris, Presses universitaires de France. 
ThÉBAud-Mony A. (2000), L'Industrie nucléaire : sous-traitance et servitude, Paris, Inserm, EDK.

Tilly L. A., Scotт J. W. (1987), Les Femmes, le travail et la famille, Paris, Rivages (édition originale en anglais publiée en 1978).

Tissot F., Messing K., Stock S. (2005), "Standing, Sitting and Associated Working Conditions in the Quebec Population in 1998”, Ergonomics, vol. 48, n 3, pp. 249-269.

TURNER A. (2014), « Corps meurtris. Genre et invalidité dans les mines de charbon d'Écosse au milieu du dix-neuvième siècle », in Rainhorn J. (dir.), Santé et travail à la mine, XIX ${ }^{e}-X X{ }^{e}$ siècle, Villeneuve-d'Ascq, Presses universitaires du Septentrion, pp. 239-260.

Vogel L. (2011), « Femmes et maladies professionnelles. Le cas de la Belgique », Rapport de l'Etui (European Trade Union Institute), nº 122.

Vogel L. (2015), « Chausser les lunettes du genre pour comprendre les conditions de travail », Hesamag, ${ }^{\circ} 12$, pp. 12-17.

Webb G. R., Redman S., Wilkinson C., SAnson-Fisher R. W. (1989), "Filtering Effects in Reporting Work Injuries”, Accident Analysis \& Prevention, vol. 21, n 2, pp. 115-123. 